\title{
Medical Image Fusion using Combined Multi-Resolution and Multi-Scaling Transform
}

\author{
Dipixa H Rana \\ M.E student of Computer Department , \\ Sigma Institute of Engineering, Vadodara
}

\begin{abstract}
Image Fusion is a process of combining the relevant information from a set of images into a single image, where the resultant fused image will be more informative and complete than any of the input images. Image fusion techniques can improve the quality. This paper uses MRI and CT images for fusion which contains complementary information helpful for diagnosis of disease. Proposed approach, medical image fusion based on the combined effect of Discrete Wavelet Transform (DWT) and Discrete Ripplet Transform (DRT). The images are initially transformed into multi-resolution image using DWT. The approximation image are further transformed using DRT. Then get ripplet coefficients and these are applied with Image Blocking method. The proposed method can be helpful for better medical diagnosis.
\end{abstract}

\section{Keywords}

Image fusion, MRI Image, CT Image, Discrete Wavelet Transform (DWT), Discrete Ripplet Transform (DRT),Image Blocking.

\section{INTRODUCTION}

Image Fusion is the process of generating better quality image from two or more input images. The resultant image should retain all important features of all input images [2]. Image fusion technology can be applied to many areas dealing with images such as medical image analysis, remote sensing, military surveillance, etc [2].

The medical imaging field demands more complementary information for disease diagnosis purpose. However, this is not possible using single modality medical images as X-ray computed tomography (CT) is suited only for recognizing bones structure, MRI giving clear information about the soft tissues and so on. In this regard, medical image fusion is the only emerging technique which has attracted researchers to assist the doctors in fusing images and retrieving relevant information from multiple modalities such as CT, MRI, FMRI, SPECT, PET [8].

Here, two input images from different image modalities are shown Fig 1 and Fig 2 .First image is a Computed Tomography (CT) image and the second image is a Magnetic Resonance Imaging (MRI). Each image has its own limitation, which can be solved by creating the fused image from two different image modalities as shown Fig 3. This would lead to improved diagnosis, better surgical planning, more accurate radiation therapy and countless other medical benefits [2]

The main advantage of Image fusion (IF) is integrating complementary, as well as redundant information from multiple images to create a fused image for providing more complete and accurate information. Another advantage of image fusion is that it reduces the storage cost by storing only the single fused image, instead of the multisource images.

\author{
Sheshang D. Degadwala \\ Assistant Professor of Computer Department , \\ Sigma Institute of Engineering, Vadodara
}

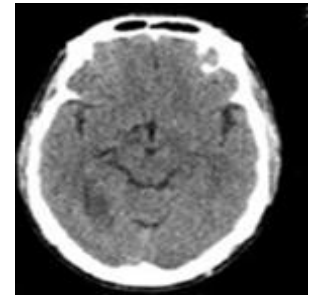

Fig 1. CT Image

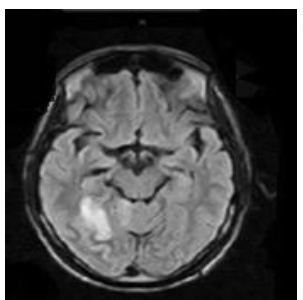

Fig 2. MRI Image

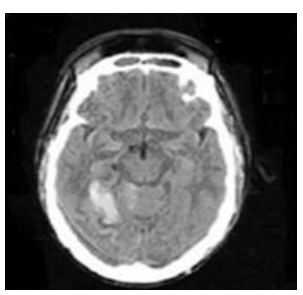

Fig 3. Fused Image

Image fusion method can be broadly classified into two groups - 1.Spatial domain fusion 2.Transform domain fusion.

Spatial image fusion methods work by combining the pixel values of the two or more images to be fused in a linear or nonlinear way [6]. This simplistic approach often has serious side effects. Pixel level image fusion methods are affected by blurring effect which directly affect on the contrast of the image [6]. The limitations of Spatial Domain are resolved by Transform Domain.

In Transform Domain, Image is first transferred into frequency domain. All the Fusion operations are performed on the Fourier transform of the image and then the Inverse Fourier transform is performed to get the resultant image [3]. Wavelet Transform is faster developed multi-resolution analysis image fusion method. The problem with Wavelet Transform (WT) is that, it can preserve spectral information efficiently but cannot express spatial characteristics well [7]. So, Recently, a theory called Multi-scale Geometric Analysis has been developed. Many MGA tools were proposed, such as Curvelet, Contourlet, Ripplet etc. Which have higher directional sensitivity [1].

In this paper, Medical Image fusion is based on combining multi-resolution transform (DWT) and multi-scaling transform (DRT). It use Image Blocking method. 
This report is organized as follows:

Section 1 gives introduction to Image Fusion, Section 2 presents description of Related Works, Section 3 presents brief description of DWT , Section 4 presents brief description of DRT, Section 5 gives Proposed algorithm of Medical Image Fusion, Section 6 gives Performance Measures parameter of Fusion techniques, finally, conclusion is presented in Section 7.

\section{RELATED WORKS}

Review of literature survey has been conducted on evaluating the performance of Medical Image Fusion Using Combined Multi-Resolution and Multi-Scaling Transform. In this section describes the previous work which had been done for Image Fusion.

C.T.Kavitha ,C. Chellamuthu, R. Rajesh, [1] proposed method using the combined effect of DWT and DRT. The DWT could detect local features of images. DRT had provide better advantage of directionality and localization, then PCCN was applied to low pass coefficients and maximum fusion rule was applied to fuse the approximation image. Proposed method had better clarity compared to DWT and PCNN methods.

Madhuri J. Patel , Keyur N. Brahmbhatt , [2] In this paper medical Image Fusion was carried out using two methods namely Contourlet transform and Contourlet Transform with Image Blocking. Image Quality \& Quantitative Metrics such as PSNR, Entropy, and NCC are used for performance evaluation. PSNR and NCC values were increase using combining Contourlet Transform and Image blocking. As each block of Fused image is original block of any of the input image visual Quality is also improved.

Madhuri J. Patel , Keyur N. Brahmbhatt, Z. H. Shah, [3] critically analyzed various Image Fusion methods and also covered What is Image Fusion overview, its major types, classification and application.

S. Das*, M. Chowdhury, and M. K. Kundu, [4] introduced the concept of Medical Image Fusion using DRT method. As a novel MGA-tool, ripplet offers better advantage of directionality, localization, multi-scale and anisotropy, which cannot be perfectly achieved by traditional MRA-tool like wavelet transform. Even though, here only used simple fusion rules in this paper, the experimental results show that RT is very effective in MIF. The proposed MIF method based on RT, is analyzed both visually and quantitatively. The proposed method is compared with CNT, and the superiority of the proposed method is established. Experimental results show that the RT based MIF, can preserve more useful information in the fused medical image with higher spatial resolution and less difference to the source images. The one disadvantage of this method is it does not provide MultiResolution.

Wang Xin , Li Yingfang, [5] Proposed method has advantage of Contourlet transform and images blocking fusion algorithms, First in this the new image fusion method combined Contourlet transform and fusion-blocking. Contourlet transform fusion algorithm with a new designed fusion rule was used for the border part between the clear area and blurring one. The source image blocks, which were more similar to the corresponding initial fused image blocks, were chosen as the final fused image blocks. Used of new algorithm effectively overcome the translation invariance of Contourlet Transform and obtains a better visual effect.
Chetan K. Solanki , Narendra M Patel, [6] In this paper two methods used for image fusion one is pixel based image fusion and another technique is wavelet based image Fusion, it uses weighted average for pixel base image fusion in this paper authors have used select maximum approach for image fusion. In this approach two input images $\mathrm{A}$ and $\mathrm{B}$ are compared with the help of pixel coordinate of images and takes only maximum value.

Second approach uses wavelet based approach. This approach reduces the blurring effect cause by pixel based method. The wavelet provides way of representing signals in time and frequency domain. This Discrete wavelet uses two types of filter high pass and low pass filter. This technique decomposes image into four images which contains different detail value based on horizontal, vertical and diagonal component. Based on this coefficient value it compares two input images and apply inverse transformation for fused image. Wavelet based fusion method offer several advantage over pixel based. It removes blurred part in output image and provide clear object then wavelet based method.

Sudeb Das and Malay Kumar Kundu Senior Member, [7] proposed multimodality MIF method based on ripplet transform using modified spatial frequency motivated PCNN. The DRT, which makes the fused images clearer and more informative. In the fused images the low frequency source subbands are fused using 'max selection' rule, and PCNN is used to select the 'better' coefficients from the decomposed source high frequency sub-bands. To improve the result, Modified spatial frequency is used as the image feature to motivate the PCNN. Results show that the more useful information in the fused image with higher spatial resolution and less difference to the source images.

Pramit parekh, Nehal Patel, Priteshkumar, Sarita Visavalia , [8] proposed different medical image fusion techniques in both spatial and transform domain have been studied. Each technique having its own Pros and Cons depends on the application. In medical imaging field, demands more information content and visualization in an image. Therefore, many medical image fusion techniques have been proposed but, still there having certain limitation. The study of different techniques of medical image fusion shows that FIS type MINSUM-MOM gives better performance in visualization perspectives then all other techniques and also give better fusion result as verified with the quantitative measures.

\section{DISCRETE WAVELET TRANSFORM (DWT)}

Discrete Wavelet Transform provide directional information in decomposition levels and contain unique information at different resolutions [1]. The fusion procedure based on wavelet transform can be described as follows.

1. The images to be fused must be registered to assure the corresponding pixels are aligned [3].

2. These images are decomposed into wavelet transformed images respectively, based on wavelet transformation. The transformed images include one low frequency portion (lowlow band) and three high frequency portions (low-high bands, high-low bands, and high-high bands) [3].

3. The transform coefficients of different portions or bands are performed with some fusion rules [3].

4. The fused image is constructed by performing an inverse wavelet transform based on the combined transform coefficients from step 3 [3]. 


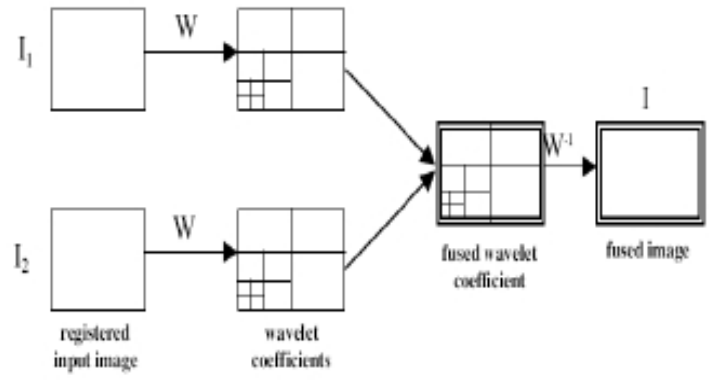

Fig.4 Block Diagram Of DWT based Image Fusion[3]

\section{DISCRETE RIPPLET TRANSFORM (DRT)}

The Conventional transforms like Fourier Transform and Wave Transform suffer from discontinuities such as edges and contours in images. To address this problem, Jun $\mathrm{Xu}$ etal. proposed a new MGA-tool called RT. The RT is a higher dimensional generalization of the Curvelet Transform (CVT), capable of representing images or $2 \mathrm{D}$ signals at different scales and different directions [4]. Visual and quantitative analysis shows, that the Ripplet Transform technique performs better compared to fusion scheme based on Contourlet Transform (CNT) [4]. RT generalizes CVT by adding two parameters, i.e., support $\mathrm{c}$ and degree d. CVT is just a special case of RT with $c=1$ and $d=2$. The anisotropy capability of representing singularities along arbitrarily shaped curves of RT is due to these new parameters $\mathrm{c}$ and $\mathrm{d}$ [4].

In DRT, $a$ is sampled at dyadic intervals. $b$ and $\theta$ are sampled at equal-spaced intervals. $a_{\mathrm{j}}, \overrightarrow{b_{k}}$ and $\theta_{\mathrm{l}}$ substitute $\mathrm{a}, \vec{b}$ and $\theta$ respectively, and satisfy that $\mathrm{a}_{\mathrm{j}}=2^{-\mathrm{j}}, \overrightarrow{b_{k}}=\left[\mathrm{c} \cdot 2^{-\mathrm{j}} \cdot \mathrm{k} 1,2^{-\mathrm{j} / \mathbf{d}}\right.$ $\left.\cdot \mathrm{k}_{2}\right]^{\mathbf{T}}$ and $\theta_{1}=2 \Pi / \mathrm{c} \cdot 2^{-[\mathrm{j}(1-1 / \mathrm{d})]} \cdot 1$, where $\vec{k}=\left[\mathrm{k}_{1}, \mathrm{k}_{2}\right]^{\mathbf{T}}$, and $\mathrm{j}, \mathrm{k}_{1}$, $k_{2}, l \in Z$. $(\cdot)^{\mathbf{T}}$ denotes the transpose of a vector. $d \in R$, since any real number can be approximated by rational numbers, so $d$ can represent as $d=n / m, n, m \neq 0 \in Z$. Usually, $n, m \in N$ and $\mathrm{n}, \mathrm{m}$ are both primes. In the frequency domain, the corresponding frequency response of ripplet function is in the form

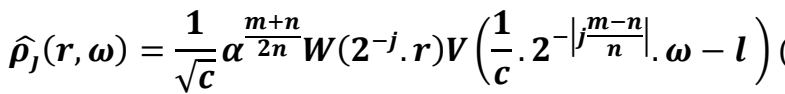

where $\mathrm{W}$ and $\mathrm{V}$ satisty the following admissibility conditions:

$$
\begin{aligned}
& \sum_{l=-\infty}^{+\infty}\left|w\left(2^{-j} \cdot r\right)\right|^{2}=1 \\
& \sum_{l=-\infty}^{\prime}\left|V\left(\frac{1}{c} 2^{-j\left(1-\frac{1}{d}\right)} \cdot \omega-l\right)\right|=1
\end{aligned}
$$

function in the frequency domain is

$$
\begin{gathered}
H_{j, l}(r, \theta)=\left\{2^{j} \leq|r| \leq 2^{2_{j}},\left|\theta-\frac{\pi}{c} \cdot 2^{-\left|j\left(1-\frac{1}{d}\right)\right|} \cdot l\right| \leq \frac{\pi}{2} 2^{-j}\right\} \\
R_{j, \vec{k}, l}=\sum_{n 1=n}^{M-1} \sum_{n ?=n}^{N-1} f(n 1, n 2) \overline{\rho_{J, \vec{k}, l(n 1 . n 2)}}
\end{gathered}
$$

$$
\widehat{f}(n 1, n 2)=\sum_{j} \sum_{\vec{k}} \sum_{l} R_{j, \vec{k}, l} \rho_{j, \vec{k}, l}(n 1, n 2)
$$

where $R_{j, \vec{k}, l}$ are the ripplet coefficients.

The image can be reconstructed through Inverse Discrete Ripplet Transform (IDRT)

\section{PROPOSED MEDICAL IMAGE FUSION ALGORITHM}

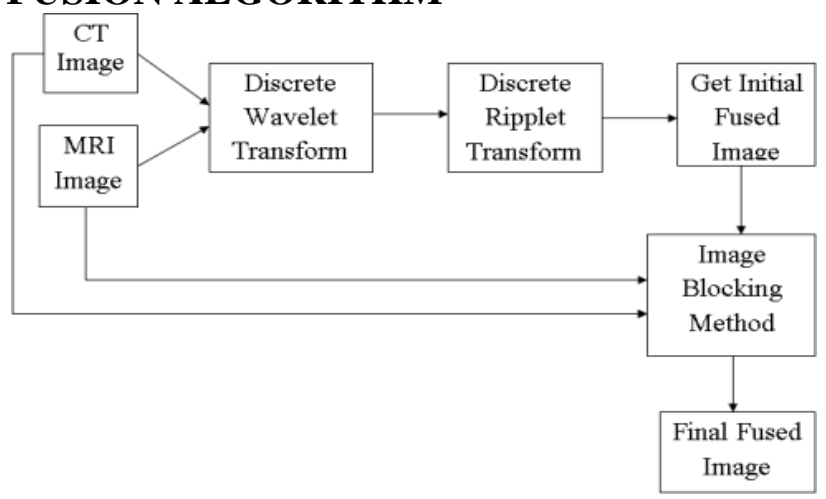

Fig.5 Block Diagram of proposed Image Fusion Method

The step can be Summarized as follows:

1. Take input images Like CT and MRI and register both these images so that the corresponding pixels are aligned means stay in the same magnitude.

2. Now apply DWT to input images, so that images are decompose into four sub-bands. These sub-bands are one low frequency sub-band and three high frequency sub-band (LL,LH, HL,HH)

3. Then get wavelet coefficients from both input images (CT,MRI) which gives high resolution and high spectral quality contents from input images.

4. After getting wavelet coefficients apply DRT transform to obtain ripplet coefficients and get initial Fused image $F_{1}$.

5. Then apply Image Blocking method. In this method, divide CT,MRI and initial fused image $F_{1}$ into equi-sized square blocks whose size are $m \times n$. Calculate the similarity measure values(SM) of the corresponding sub-blocks of CT, MRI and $F_{1}$ respectively.

$$
\begin{gathered}
S M_{F_{1} M R I}=\frac{2 \times \sum_{i=1}^{m} \sum_{i=1}^{n} F_{1}(i, j) \times M R I(i, j)}{\sum_{i=1}^{m} \sum_{i=1}^{n}\left[F_{1}(i, j)^{2}+M R I(i, j)^{2}\right]} \\
S M_{F_{1} C T}=\frac{2 \times \sum_{i=1}^{m} \sum_{i=1}^{n} F_{1}(i, j) \times C T(i, j)}{\sum_{i=1}^{m} \sum_{i=1}^{n}\left[F_{1}(i, j)^{2}+C T(i, j)^{2}\right]}
\end{gathered}
$$

The higher the value of SM indicates the high similarity of two images. If the similarity between the source image block and the initial fused image block is greater, the greater the probability is that the ultimate fused image blocks comes from the source image block. 
6. Create Matrix $S(r, c)$ :

$$
\mathrm{S}(\mathrm{r}, \mathrm{c})=\left\{\begin{array}{cc}
1 S M_{F_{1} C T} & (r, c) \geq S M_{F_{1} M R I}(r, c) \\
0 & \text { otherwise }
\end{array}\right.
$$

where $\mathrm{M}, \mathrm{N}$ is the length and width of the source image. $\mathrm{r}=1,2, \ldots, \mathrm{M} / \mathrm{m}$ and $\mathrm{c}=1,2, \ldots, \mathrm{N} / \mathrm{n} . \mathrm{r}$ and $\mathrm{c}$ are actually the image block position coordinates in the image.

Check consistency on the $\mathrm{S}$ matrix, then get the final fused image $\mathrm{F}$ by the following calculation:

If $S(r, c)=1$ and the sum of each element in the $S$ matrix entered at $(r, c)$ of $3 \times 3$ neighborhood is equal to 9 , then:

$\mathbf{F}(\mathbf{i}, \mathbf{j})=F_{C T}(\mathbf{i}, \mathbf{j})$

Else if: $\mathrm{S}(\mathrm{r}, \mathrm{c})=0$ and the sum of each element in the $\mathrm{S}$ matrix centered at $(r, c)$ of $3 \times 3$ neighborhood is equal to 0 , then:

$\mathbf{F}(\mathbf{i}, \mathbf{j})=F_{M R I}(\mathbf{i}, \mathbf{j})$

else :

$F(\mathbf{i}, \mathbf{j})=F_{1}(\mathbf{i}, \mathbf{j})$

And finally get fused Image.

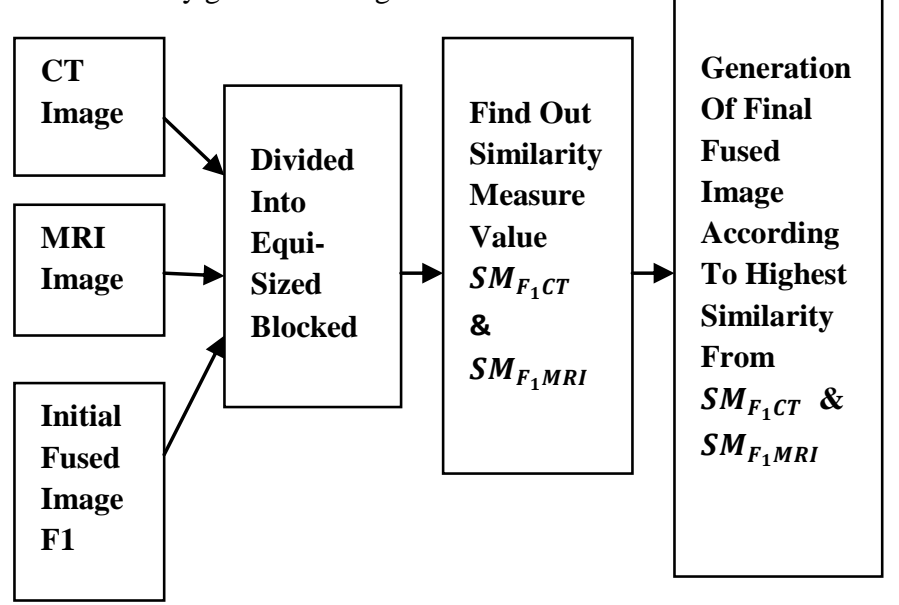

Fig.6 Block Diagram of Image Blocking Method

\section{PERFORMANCE MEASURES PARAM -ETER OF FUSION TECHNIQUES}

\subsection{Entropy:}

Entropy is one of the most important quantitative measures in image fusion. A digital image consists of pixels arranged in rows and columns. Each pixel in Image is defined by its position and gray scale level. For an image consists of L gray levels, the entropy is defined as [8]:

$$
E=-\sum_{i=0}^{L-1} p_{i} \log _{2} p_{i}
$$

Where, $\mathrm{P}(\mathrm{i})$ is probability of each grey scale level.

\subsection{Peak Signal To Noise Ratio(PSNR)}

Peak Signal To Noise Ratio [2] can be given as follow:

$$
P S N R=10 \times \log _{10}\left(\frac{f_{\max }^{2}}{M S E}\right)
$$

Where, $f_{\max }$ is maximum gray scale value of the pixels in fused image, the higher the value of the PSNR, the better the fusion result.

\subsection{Normalized Cross Correlation(NCC)}

It used to find out similarities between fused image and registered image is given by the following equation [2]:

$$
N C C=\sum_{i=1}^{m} \sum_{i=1}^{n}\left(F_{i j} \times R_{i j}\right) / \sum_{i=1}^{m} \sum_{i=1}^{n} F_{i j}^{2}
$$

Where, $\mathrm{R}$ is original image (or one of the source images) and $\mathrm{F}$ is the fused image. $\mathrm{m}$ and $\mathrm{n}$ are the dimensions of the images.

\section{CONCLUSION}

The proposed image fusion method based on combination of Discrete Wavelet Transform and Discrete Ripplet Transform can works efficiently for fusion of medical imaging applications. The Quality and Quantity of Fused Image can be can be measured based on the PSNR value ,Entropy ,NCC. PSNR value must be increase, the higher the value of the PSNR means the better fusion result.

\section{REFERENCES}

[1] C.T . Kavitha, C. Chellamuthu, R. Rajesh, "Medical Image Fusion using combined wavelet and ripplet transforms" Elsevier, 2012.

[2] Madhuri J. Patel, Keyur N. Brahmbhatt, "Medical Image Fusion" International journal of Advance Engineering and Research development (IJAERD), 2014, vol, no.1, Issue 5.

[3] Madhuri J. Patel, Keyur N. Brahmbhatt, Z. H. Shah, "Comparative Study on Image Fusion Methods" ,International Journal of Graphics \& Image Processing, Vol.4,issue 1,Feb. 2014.

[4] S. Das*, M. Chowdhury, and M. K. Kundu, "Medical Image Fusion Based On Ripplet Transform Type-I", Progress In Electromagnetics Research B, Vol. 30, 355-370, 2011.

[5] Wang Xin, Li Yingfang , "A New Method for Multi-Focus Image Fusion Using Countourlet Transform",IEEE, 2011.

[6] Chetan K. Solanki, Narendra M Patel , "Pixel Based And Wavelet Based Image Fusion Methods With Their Comparative Study" NCRTET, 2011.

[7] Sudeb Das, Malay Kumar Senior Member, "Ripplet Based Multimodality Medical Image Fusion using Pulse Coupled Neurzl Network and Modified Spatial Frequency" IEEE, 2011.

[8] Pramit Parekh, Nehal Patel, Priteshkumar, Sarita Visavalia, "Comparative Study and Analysis of Medical Image Fusion Techniques" (IJCA),2014. 